\title{
On the (In)security of Fischlin's Paradigm
}

\author{
Prabhanjan Ananth ${ }^{1}$, Raghav Bhaskar ${ }^{1}$, Vipul Goyal ${ }^{1}$, and Vanishree Rao ${ }^{2}$ \\ 1 Microsoft Research India \\ prabhanjan.va@gmail.com, \{rbhaskar,vipul\}@microsoft.com \\ 2 Department of Computer Science, UCLA \\ vanishri@cs.ucla.edu
}

\begin{abstract}
The Fiat-Shamir paradigm was proposed as a way to remove interaction from 3-round proof of knowledge protocols and derive secure signature schemes. This generic transformation leads to very efficient schemes and has thus grown quite popular. However, this transformation is proven secure only in the random oracle model. In FOCS 2003, Goldwasser and Kalai showed that this transformation is provably insecure in the standard model by presenting a counterexample of a 3-round protocol, the Fiat-Shamir transformation of which is (although provably secure in the random oracle model) insecure in the standard model, thus showing that the random oracle is uninstantiable. In particular, for every hash function that is used to replace the random oracle, the resulting signature scheme is existentially forgeable. This result was shown by relying on the non-black-box techniques of Barak (FOCS 2001).

An alternative to the Fiat-Shamir paradigm was proposed by Fischlin in Crypto 2005. Fischlin's transformation can be applied to any so called 3-round "Fiat-Shamir proof of knowledge" and can be used to derive non-interactive zero-knowledge proofs of knowledge as well as signature schemes. An attractive property of this transformation is that it provides online extractability (i.e., the extractor works without having to rewind the prover). Fischlin remarks that in comparison to the Fiat-Shamir transformation, his construction tries to "decouple the hash function from the protocol flow" and hence, the counterexample in the work of Goldwaaser and Kalai does not seem to carry over to this setting.

In this work, we show a counterexample to the Fischlin's transformation. In particular, we construct a 3-round Fiat-Shamir proof of knowledge (on which Fischlin's transformation is applicable), and then, present an adversary against both - the soundness of the resulting non-interactive zero-knowledge, as well as the unforegeability of the resulting signature scheme. Our attacks are successful except with negligible probability for any hash function, that is used to instantiate the random oracle, provided that there is an apriori (polynomial) bound on the running time of the hash function. By choosing the right bound, secure instantiation of Fischlin transformation with most practical cryptographic hash functions can be ruled out.

The techniques used in our work are quite unrelated to the ones used in the work of Goldwasser and Kalai. Our primary technique is to bind the protocol flow with the hash function if the code of the hash function is available. We believe that our ideas are of independent interest and maybe applicable in other related settings.
\end{abstract}




\section{Introduction}

The Fiat-Shamir paradigm [FS86] was proposed as a way to remove interaction from 3-round proof of knowledge protocols and derive secure signature schemes. It is a generic transformation which leads to quite efficient schemes thus making it quite popular. The security of this transformation was later analyzed under the ideal assumption that the hash function behaves as a random oracle BR93, PS00]. Thus, the resulting non-interactive proofs and signature scheme are automatically secure in the random oracle model. Several signature schemes (with the best known ones being [Sch91, GQ88, Oka92]) were constructed following the Fiat-Shamir paradigm. It has also been useful in obtaining forward secure schemes and improving the tightness of security reductions [AABN02, MR02.

Random oracle model can be seen as a methodology to design secure cryptographic systems in two steps: first construct and analyze a scheme assuming only oracle access to the random function. Then, find a suitable hash function and instantiate the previous construction with that to get a real world secure cryptographic system.

For the Fiat-Shamir paradigm, Goldwasser and Kalai GK03 showed that unfortunately the second step of the design methodology cannot be carried out. In particular, they show that the Fiat-Shamir transformation is uninstantiable in the real world: regardless of the choice of the hash function, the resulting signature scheme is insecure. To do this, they first gave a construction of a 3round identification scheme based on the non-black-box simulation techniques of Barak Bar01, and then showed that the resulting signature scheme is universally forgeable for any hash function.

An alternative to the Fiat-Shamir paradigm was proposed by Fischlin [Fis05]. Fischlin's transformation can be applied to any so called 3-round "Fiat-Shamir proof of knowledge" and can be used to derive non-interactive zero-knowledge proofs of knowledge as well as signature schemes. An attractive property of this transformation is that it provides online extractability. In other words, just by observing queries a (possibly malicious) prover makes to the random oracle, an extractor is guaranteed to be able to output the witness of the statement being proven (except with negligible probability). This is in contrast to Fiat-Shamir transformation where an extractor must work by rewinding the prover. This property is quite useful while using the resulting non-interactive schemes in larger systems. Fischlin also shows applications of his transformation in constructing group signature schemes [BBS04.

Even though the purpose of Fischlin's transformation is quite similar to that of Fiat-Shamir, the transformation itself is quite different. Fiat-Shamir transformation is applied on a 3 round public coin proof of knowledge protocol and works as follows. Prover sends a commitment to the verifier, the verifier sends back a random challenge, and, the prover finally responds to that challenge. In the transformed non-interactive protocol, the challenge of the verifier is generated by applying the random oracle to the first message (i.e., the commitment) of the prover. The non-interactive scheme is secure in the random oracle model since getting the challenge from the random oracle is similar to getting it from 
the external verifier; both challenges will be unpredictable to a malicious prover and trying again any polynomial number of times does not help.

Goldwasser and Kalai GK03 showed insecurity of the Fiat-Shamir paradigm by relying the breakthrough work of Barak Bar01. Indeed it seems (in retrospect) that the non-black-box simulation techniques of Barak fits in quite well to show insecurity of the Fiat-Shamir paradigm:

- In the Fiat-Shamir paradigm, the verifier basically just acts as a hash function (i.e., the verifier message is computed by simply evaluating the hash function on the incoming prover message).

- Hence, having oracle access to the hash function is similar to having a blackbox access to the verifier, while, having the code of the hash function directly translates to having non-black-box access to the verifier.

- Barak's techniques yield a zero-knowledge protocol which is secure given only black-box access to the verifier (in other words, a scheme which is resettablysound [BGGL01]), but becomes insecure given non-black-box access to the verifier.

We remark that even though the above idea is the starting point towards showing insecurity of the Fiat-Shamir paradigm in GK03, this by itself is not sufficient. This is because Barak's techniques do not yield a 3-round protocol. Goldwasser and Kalai make use of a number of additional tools and ideas; please refer to GK03 for more details.

The above high level idea completely breaks down in the context of Fischlin's transformation. As Fischlin remarks [Fis05, "in comparison to the Fiat-Shamir transformation, this construction somewhat decouples the hash function from the protocol flow". In other words, the prover and the verifier messages of the underlying scheme are computed as specified in the underlying scheme; not by making use of the hash function in any way. The hash function is only used to make some final checks on the resulting transcript of interaction. Hence, as observed by Fischlin, the counterexample in GK03 does not seem to carry over to this setting Fis05. This raises the following natural question.

"Is there a concrete hash function using which Fischlin transformation can be securely instantiated?"

Our Results. In this work, we give a partial answer to the above question. More specifically, we prove the following.

"There does not exist any hash function, whose running time is bounded by an apriori fixed polynomial, using which Fischlin transformation can be securely instantiated."

One can interpret the above result in two different ways. Firstly, the bound on the running time will typically be chosen to be a large polynomial in the security parameter. By choosing a large bound, we can rule out the instantiation of Fischlin transformation with widely used hash functions such as SHA-1. Another interpretation is that, given any hash function, we can construct a (3-round 
Fiat-Shamir proof of knowledge) protocol such the the following holds. When Fischlin's transformation is applied on this protocol and instantiated using this hash function, the resulting signature scheme as well the non-interactive zeroknowledge scheme is completely insecure.

We note that the above does not invalidate the original security proof of Fischlin's transformation in any way (which are provided only in the random oracle model). No claims regarding the security of the transformation, once the hash function is instantiated are made in [Fis05]. Fischlin explicitly acknowledges the possibility of such a result in the introduction of his paper [Fis05].

\subsection{Technical Overview}

Before we discuss the techniques involved in our work, we briefly sketch Fischlin's transformation in the following.

Fischlin's Transformation. Fischlin [Fis05] proposed an approach to transform any Fiat-Shamir proof of knowledge (as defined in Section 2) to a non-interactive zero knowledge proof of knowledge in the random oracle model. The basic idea of his transformation is given in the following. The transformed prover (of the non-interactive zero-knowledge scheme) roughly works as follows.

- In the underlying Fiat-Shamir proof of knowledge, the challenge space is restricted to be of polynomial size (i.e., the challenge string of the verifier will be of logarithmic length). The protocol begins by executing a superconstant number of parallel copies of the honest prover of the underlying Fiat-Shamir proof of knowledge.

- For each parallel execution $i$, the prover computes the commitment $\alpha_{i}$ (i.e., the first message).

- For all possible (polynomially many) challenges $\beta_{i}$ starting from 0 the prover performs the following test. It checks whether a fixed number (depending on the security parameter) of least significant bits of $\mathcal{O}\left(\left(\alpha_{1}, \ldots, \alpha_{r}\right), i, \beta_{i}, \gamma_{i}\right)$ are all 0 . Here $\gamma_{i}$ is the prover's response to the challenge $\beta_{i}$ and $\mathcal{O}$ denotes the random oracle. If the test passes, the prover fixes $\beta_{i}$ to be the challenge for session $i$.

- Finally, the transcript $\left(\alpha_{i}, \beta_{i}, \gamma_{i}\right)$ corresponding to every execution $i$ is output as the proof.

- Verifier accepts the proof only if: (a) the transcript for every execution is accepted by the verifier of the underlying Fiat-Shamir proof of knowledge, and, (b) for all executions, the least significant bits of the random oracle invocation come out to be all 0 (as described above). 1

The above construction retains the completeness property: except with negligible probability, for each execution, there will be at least one challenge $\beta_{i}$ for which the random oracle outputs 0 (in the relevant bits).

${ }^{1}$ This is actually the simplified version of the final construction in [Fis05]. Our results apply to both the variants. 
The construction provides soundness for the following reasons. If the statement is false, for each $\alpha_{i}$, there is a single challenge $\beta_{i}$ for which a satisfying response $\gamma_{i}$ can be given. Consider the vector of first messages chosen by the adversary $\left(\alpha_{1}, \ldots, \alpha_{r}\right)$. Except with negligible probability, there will exist at least one $i$ such that $\mathcal{O}\left(\left(\alpha_{1}, \ldots, \alpha_{r}\right), i, \beta_{i}, \gamma_{i}\right)$ does not have its required bits to be all 0 . Once that happens, the adversary will have to change $\alpha_{i}$ (and hence the vector $\left(\alpha_{1}, \ldots, \alpha_{r}\right)$ changes). Thus, adversary has to essentially restart its effort to produce a false proof (and again it will only be successful with negligible probability).

Thus, it is crucial to have the entire vector $\left(\alpha_{1}, \ldots, \alpha_{r}\right)$ as part of the input to the random oracle. Even if the adversary fails to obtain the required 0's even in a single execution, it has to start again from scratch. See section 2 for more details.

Our Ideas. Recall that the verifier accepts the proof only if: (a) the transcript for every execution is accepted by the verifier of the underlying Fiat-Shamir proof of knowledge, and, (b) for all executions, the least significant bits of the random oracle invocation come out to be all 0 . Normally, these two tests will be "independent and uncorrelated". This is because no random oracle invocations are involved in the first test (the underlying Fiat-Shamir proof of knowledge protocol does not make use of random oracles). However once the code of the hash function is available, it can be used in the underlying protocol making the two tests correlated. In fact, in our construction, the two tests will end up being identical. This would allow an adversarial prover to succeed (using the description of the hash function). Below we provide a very high level overview of our main idea.

- Observe that in the final transcript being output, for each session $i$, adversary needs to include an accepting response for just a single challenge $\beta_{i}$ (for which the random oracle output has 0 in all required positions). What if somehow magically, adversary exactly has the capability to come up with an accepting response for just this $\beta_{i}$ (note that adversary can have the capability of creating an accepting response just for a single challenge)?

- To achieve the following, we take any Fiat-Shamir proof of knowledge and then add another "mode of execution" to it. In this mode, the prover doesn't need the witness to execute the protocol. However the verifier's test is such that for each $\alpha_{i}$, there is a single $\beta_{i}$ for which verifier accepts. Hence, the protocol still maintains the special soundness property. This new protocol will be the underlying protocol on which Fischlin's transformation will be applied.

- Now we sketch the test the verifier performs in this second mode. The prover will be free to send any hash function to the verifier as part of $\alpha_{i}$. Using this hash function, the verifier is instructed to compute the only acceptable $\beta_{i}$ for this $\alpha_{i}$. If that is the challenge he chose, the verifier is instructed to accept (and reject otherwise). 
- The acceptable $\beta_{i}$ is the first possible challenge (lexicographically) such that $H\left(\left(\alpha_{1}, \ldots, \alpha_{i}, \ldots \alpha_{r}\right), i, \beta_{i}, \gamma_{i}\right)$ has 0 in all the required positions (where $H$ is the hash function chosen by the prover).

- Now if the hash function $H$ is the same as the random oracle, we have that the second test (by the verifier of the non-interactive proof) is satisfied for free. Hence, by running in the second mode, soundness of the non-interactive scheme can be violated.

There are several problems with this basic idea. To start with, the verifier of the (interactive) Fiat-Shamir protocol that we constructed is unaware of any other sessions. Whether or not it accepts should only be decided by the transcript of this session. However the test $H\left(\left(\alpha_{1}, \ldots, \alpha_{i}, \ldots \alpha_{r}\right), i, \beta_{i}, \gamma_{i}\right)$ requires the knowledge of the first prover messages in all other sessions (we resolve this problem by having a construction in which a first prover message for one session can be used to compute first prover messages for the other sessions). Secondly, note that the random oracle instantiation could be done by any (possibly adversarial) hash function. Since the transcript of interaction in mode 1 and mode 2 may be easily distinguishable, the hash function may never give output having 0 in the relevant places for mode 2 messages (we solve this problem by employing encryption in a deterministic way using shared public randomness). The final construction is given in Section 3 ,

We note that once we have an adversary to violate soundness of the noninteractive zero-knowledge scheme, it is also easy to design a forger for the resulting signature scheme.

Further Comments. We note that Fischlin's transformation could still be secure in the following sense. For every protocol (on which Fischlin's transformation can be applied), there exists a hash function, whose running time depends upon the parameters of the protocol (in particular upon the running time of the parties in the protocol) such that the following happens. The signature scheme (and non-interactive zero-knowledge scheme) obtained by applying Fischlin's transformation on this protocol, when instantiated with this hash function, is secure in the plain model. However we note that the hash function used to instantiate the scheme has to be dependent on the protocol. In particular, one cannot use a fixed hash function (such as SHA-256) to instantiate the resulting schemes.

Furthermore, Fischlin's construction could still be instantiated if there are no shared public parameters between the prover and the verifier. As with the counterexample for the Fiat-Shamir transformation GK03, our construction is in the setting where the prover and the verifier share some public parameter:2. We also sketch an extension of our main construction to the setting where the prover and the verifier have no prior communication/setup in Section 4 In this setting, our results are only valid for the class of hash function whose output is pseudorandom. Indeed, it is natural to think of the random oracle being instantiated by a pseudorandom function. We leave getting an unrestricted result in this setting as an open problem.

\footnotetext{
${ }^{2}$ Note that none of the parties need to trust the public parameters for their security.
} 
Related Works. A number of works have investigated the difference in the settings: where one only has oracle access to a primitive $\mathrm{v} / \mathrm{s}$ having the full code of the primitive. These lines of research include ones on program obfuscation $B G I^{+} 01$, GK05, non-black-box simulation techniques [Bar01, Pas04, DGS09, uninstantiabilty of constructions in the random oracle model [CGH04, etc.

\section{Fischlin Transformation}

In this section, we shall review the Fischlin transformation. We begin by stating the preliminaries. Throughout the paper, we denote the security parameter by $k$. A function $f: \mathbb{N} \rightarrow \mathbb{R}^{+} \cup\{0\}$ is said to be negligible (in its input) if, for any constant $c>0$, there exists a constant, $k_{0} \in \mathbb{N}$, such that $f(k)<(1 / k)^{c}$ for any $k>k_{0}$. We let $f(k)=n e g l(k)$ denote that $f(k)$ is a negligible function. We say that function is non-negligible if it is not negligible; namely, we say that $f(\cdot)$ is non-negligible in its input if there is constant $c i, 0$ such that for infinitely many $k$, it holds $f(k) \geq(1 / k)^{c}$. For a probabilistic polynomial time algorithm $\mathcal{A}$, we use the notation $y \leftarrow \mathcal{A}(x)$ to denote $\mathcal{A}$ outputting $y$ on input $x$. We use the notation $\operatorname{Pr}[E] \gtrsim 1$ to indicate that the probability of the event $E$ is negligibly close to 1 . Similarly, the notation $\operatorname{Pr}[E] \gtrsim 0$ is used to indicate that the probability of the event $E$ is neglibly close to 0 .

In this paper, we scrutinize the "real-world" security of protocols that are proven secure in the random oracle model. In the random oracle model, all the parties have access to a purely random function (i.e., a mapping that maps every input to an output that is distributed uniformly random in a range space whose size is dependent on the security parameter. We denote the random oracle by $\mathcal{O}$.

Fischlin transformation converts a 3-round zero-knowledge proof of knowledge, termed as Fiat-Shamir proof of knowledge, to a non-interactive zeroknowledge proof of knowledge proven secure in the random oracle model. In what follows we shall review the formal definitions for both Fiat-Shamir proof of knowledge as well as non-interactive zero-knowledge proof of knowledge as defined in Fis05.

Definition 1. A Fiat-Shamir proof of knowledge (with $O(\log (k))$-bit challenges) for a witness relation $W$ is a pair $(P, V)$ of probabilistic polynomial time algorithms $P=\left(P_{0}, P_{1}\right), V=\left(V_{0}, V_{1}\right)$ with the following properties.

[Completeness]. For any parameter $k,(x, w) \in W_{k},(\alpha, \beta, \gamma) \leftarrow\left(P(x, w), V_{0}(x)\right)$ it holds $V_{1}(x, \alpha, \beta, \gamma)=$ Accept.

[Commitment Entropy]. For parameter $k$, for any $(x, w) \in W_{k}$, the min-entropy of $\alpha \leftarrow P_{0}(x, w)$ is superlogarithmic in $k$.

[Public Coin]. For any $k$, any $(x, w) \in W_{k}$, any $\alpha \leftarrow P_{0}(x, w)$, the challenge $\beta \leftarrow V_{0}(x, \alpha)$ is uniform on $\{0,1\}^{l(k)}$. 
[Unique responses]. For any probabilistic polynomial time algorithm A, for parameter $k$ and $\left(x, \alpha, \beta, \gamma, \gamma^{\prime}\right) \leftarrow A(k)$, we have, as a function of $k$,

$$
\operatorname{Pr}\left[V_{1}(x, \alpha, \beta, \gamma)=V_{1}\left(x, \alpha, \beta, \gamma^{\prime}\right)=\text { Accept } \wedge \gamma \neq \gamma^{\prime}\right] \approx 0
$$

[Special Soundness]. There exists a probabilistic polynomial time algorithm $K$, the knowledge extractor, such that for any $k$, any $(x, w) \in W_{k}$, any pairs $(\alpha, \beta, \gamma)$, $\left(\alpha, \beta^{\prime}, \gamma^{\prime}\right)$ with $V_{1}(x, \alpha, \beta, \gamma)=V_{1}\left(x, \alpha, \beta^{\prime}, \gamma^{\prime}\right)=$ Accept and $\beta \neq \beta^{\prime}$, for $w^{\prime} \leftarrow$ $K\left(x, \alpha, \beta, \gamma, \beta^{\prime}, \gamma^{\prime}\right)$ it holds $\left(x, w^{\prime}\right) \in W_{k}$.

[Honest-Verifier Zero-Knowledge]. There exists a probabilistic polynomial time algorithm $Z$, the zero-knowledge simulator, such that for any pair of probabilistic polynomial time algorithms $D=\left(D_{0}, D_{1}\right)$ the following distributions are computationally indistinguishable:

- Let $(x, w, \delta) \leftarrow D_{0}(k)$ and $(\alpha, \beta, \gamma) \leftarrow\left(P(x, w), V_{0}(x)\right)$ if $(x, w) \in W_{k}$ and $(\alpha, \beta, \gamma) \leftarrow \perp$ otherwise. Output $D_{1}(\alpha, \beta, \gamma, \delta)$.

- Let $(x, w, \delta) \leftarrow D_{0}(k)$ and $(\alpha, \beta, \gamma) \leftarrow Z(x, \mathrm{YES})$ if $(x, w) \in W_{k}$ and $(\alpha, \beta, \gamma)$ $\leftarrow Z(x, \mathrm{NO})$. Output $D_{1}(\alpha, \beta, \gamma, \delta)$.

Definition 2. A pair $(P, V)$ of probabilistic polynomial time algorithms is called a non-interactive zero-knowledge proof of knowledge for relation $W$ with an online extractor (in the random oracle model) if the following holds.

[Completeness] For any oracle $\mathcal{O}$, any $(x, w) \in W_{k}$ and any $\pi \leftarrow P^{\mathcal{O}}(x, w)$, we have $\operatorname{Pr}\left[V^{\mathcal{O}}(x, \pi)=\right.$ Accept $] \gtrsim 1$.

[Zero-knowledge] There exist a pair of probabilistic polynomial time algorithms $Z=\left(Z_{0}, Z_{1}\right)$, the zero-knowledge simulator, such that for any pair of probabilistic polynomial time algorithms $D=\left(D_{0}, D_{1}\right)$, the following distributions are computationally indistinguishable:

- Let $\mathcal{O}$ be a random oracle, $(x, w, \delta) \leftarrow D_{0}^{\mathcal{O}}(k)$, and $\pi \leftarrow P^{\mathcal{O}}(x, w)$ where $(x, w) \in W_{k}$. Output $D_{1}^{\mathcal{O}}(\pi, \delta)$.

- Let $\left(\mathcal{O}_{0}, \sigma\right) \leftarrow Z_{0}(k),(x, w, \delta) \leftarrow D_{0}^{\mathcal{O}_{0}}(k)$ and $\left(\mathcal{O}_{1}, \pi\right) \leftarrow Z_{1}(\sigma, x)$. Output $D_{1}^{\mathcal{O}_{1}}(\pi, \delta)$.

[Online Extractor]. There exists a probabilistic polynomial time algorithm $K$, the online extractor, such that the following holds for any algorithm $A$. Let $\mathcal{O}$ be a random oracle, $(x, \pi) \leftarrow A^{\mathcal{O}}(k)$ and $Q_{\mathcal{O}}(A)$ be the sequence of queries of $A$ to $\mathcal{O}$ and $\mathcal{O}$ 's answers. Let $w \leftarrow K\left(x, \pi, Q_{\mathcal{O}}(A)\right)$. Then, as a function of $k$,

$$
\operatorname{Pr}\left[(x, w) \notin W_{k} \wedge V^{\mathcal{O}}(x, \pi)=\text { Accept }\right] \approx 0
$$

We are now ready to give a formal description of the Fischlin transformation.

Fischlin Transformation. Let $\left(P_{F S}, V_{F S}\right)$ be an interactive Fiat-Shamir proof of knowledge with challenges of $l=l(k)=O(\log (k))$ bits for a relation $W$. Define the parameters $b, r, S, t$ as the number of test bits, repetitions, maximum 
sum and trial bits such that $b r=\omega(\log (k)), t-b=\omega(\log (k)), b, r, t=O(\log (k))$, $S=O(r)$ and $b \leq t \leq l$. Define the following non-interactive proof system for relation $W$ in the random oracle model where the random oracle maps to $b$ bits.

Prover: The prover $P^{\mathcal{O}}$ on input $(x, w)$, first runs the prover $P_{F S}(x, w)$ in $r$ independent repetitions to obtain $r$ commitments $\left(\alpha_{1}, \ldots, \alpha_{r}\right)$. Then $P^{\mathcal{O}}$ does the following, either sequentially or in parallel for each repetition $i$. For each $\beta_{i}=0,1, \ldots, 2^{t}-1$ it lets $P_{F S}$ compute the final responses $\gamma_{i}$ by rewinding, until it finds the first one such that $\mathcal{O}\left(x,\left(\alpha_{1}, \ldots, \alpha_{r}\right), i, \beta_{i}, \gamma_{i}\right)=0^{b}$, if no such tuple is found then $P^{\mathcal{O}}$ picks the first one for which the hash value is minimal among all $2^{t}$ hash values. The prover finally outputs $\pi=\left(\alpha_{i}, \beta_{i}, \gamma_{i}\right)_{i=1, \ldots, r}$.

Verifier: The verifier $V^{\mathcal{O}}$ on input $x$ and $\pi=\left(\alpha_{i}, \beta_{i}, \gamma_{i}\right)_{i=1, \ldots, r}$ accepts if and only if $V_{1, F S}\left(x, \alpha_{i}, \beta_{i}, \gamma_{i}\right)=$ Accept (first test) for each $i \in[r]$ and if $\sum_{i=1}^{r} \mathcal{O}\left(x,\left(\alpha_{1}, \ldots, \alpha_{r}\right), i, \beta_{i}, \gamma_{i}\right) \leq S$ (second test $)$.

We shall now briefly review the proof of security (in the random oracle model) of the Fischlin transformation. We shall begin by arguing completeness. We need to show that the (honest) prover fails to convince the verifier only with negligible probability. From the completeness property of the underlying Fiat-Shamir proof of knowledge, the proof produced by the honest prover passes the first test with probability 1 . It can be shown that the probability that the proof passes the second test is negligibly close to 1 by the following two basic arguments:

- Probability that at least in one of the $r$ repititions the smallest hash value that the prover obtains is $>S$ is negligible. Hence, with all but negligible probability the sum of the hash values $\leq r S$.

- By a basic combinatorial argument, the sum of the hash values $>S$ and $\leq r S$ only with negligible probability. Hence, the sum is $\leq S$ with all but negligible probability.

From this, it can be seen that the honest prover passes the second test with probability negligibly close to 1 .

We now prove that the protocol satisfies online extractability (which in turn implies soundness). Consider an adversarial prover who produces a proof given just the input instance. The claim is that except with negligible probability the proof is rejected by the verifier. Consider a particular commitment tuple $\left(\alpha_{1}, \ldots, \alpha_{r}\right)$. Observe that in the queries made by the adversarial prover to the random oracle there cannot be two accepting transcripts of the form $\left(\left(\alpha_{1}, \ldots, \alpha_{r}\right), i, \beta, \gamma\right)$ and $\left(\left(\alpha_{1}, \ldots, \alpha_{r}\right), i, \beta^{\prime}, \gamma^{\prime}\right)$ because then the special soundness property of the underlying Fiat-Shamir proof of knowledge would imply that the adversary has the witness for the input instance. Hence, corresponding to each repetition $i$ and commitment tuple $\left(\alpha_{1}, \ldots, \alpha_{r}\right)$, the adversary can query the random oracle for at most one challenge $\beta_{i}$. Let $s_{i}$ be the value output by the random oracle for this particular $\beta_{i}$. With negligible probability, the summation of $s_{i}$ over all the repetitions is at most $S$. This is because, there are only polynomially many (in the security parameter) possible tuples $\left(s_{1}^{\prime}, \ldots, s_{r}^{\prime}\right)$ whose 
summation is at most $S$ and since $s_{i}$ is picked uniformly at random, the probability that the sum of $s_{i}$ is at most $S$ is $\operatorname{poly}(k) . n e g l(k)$ which is also negligible in $k$. This means that the adversary has negligible probability of succeeding for a given $\left(\alpha_{1}, \ldots, \alpha_{r}\right)$. Since, the adversary can try only polynomially many such commitment tuples, it is only with negligible probability that it can produce an accepting proof.

Fischlin showed that the non-interactive zero-knowledge proof of knowledge obtained from his construction can be used to construct signature schemes which are secure. The signature scheme derived from his construction was shown to be existentially unforgeable against adaptive chosen message attacks in the random oracle model.

In this paper, we give a construction of 3-round Fiat-Shamir proof of knowledge protocol such that the resulting protocol obtained after applying Fischlin transformation does not satisfy soundness in the real-world. And hence, note that the security of the signature scheme built over this non-interactive protocol (which is the output of Fischlin transformation) also breaks down.

\section{Our Construction}

Our goal is to construct a Fiat-Shamir proof of knowledge $\left(P^{*}, V^{*}\right)$ for some witness relation such that the non-interactive protocol obtained after applying the Fischlin transformation to it is insecure when the random oracle is instantiated with a hash function ensemble containing functions whose running time is apriori bounded by some polynomial. We now give the intuition about the construction of proof of knowledge $\left(P^{*}, V^{*}\right)$. We first consider a Fiat-Shamir proof of knowledge $(P, V)$ from which we build $\left(P^{*}, V^{*}\right)$. The verifier $V^{*}$ is basically $V$ with its verdict function extended so as to also accept in the case when the challenge equals the output of some pre-determined function, denoted by least (defined later). The function least takes the first message as input and returns a challenge. As the verifier chooses the challenge uniformly at random, it is only with low probability that the challenge equals the output of least of the first message. This, together with the fact that $(P, V)$ is sound, implies that $\left(P^{*}, V^{*}\right)$ satisfies soundness property. However, in the non-interactive protocol obtained by applying Fischlin transformation to $\left(P^{*}, V^{*}\right)$, denoted by $\left(P^{\mathcal{O}}, V^{\mathcal{O}}\right)$, the prover himself is supposed to compute the challenge messages. However, the probability that any adversarial prover succeeds in producing an accepting proof is negligible from the security proof of the Fischlin transformation. But when the random oracle $\mathcal{O}$ in $\left(P^{\mathcal{O}}, V^{\mathcal{O}}\right)$ is instantiated by a hash function $h$ drawn from a hash function ensemble to get $\left(P_{h}, V_{h}\right)$ we can construct an adversary to violate the soundness of $\left(P_{h}, V_{h}\right)$ as follows. The adversarial prover first makes the least function dependent on machine $\mathrm{M}$, which implements the instantiated hash function $h$, in such a way that the first test and the second test become identical. The adversary then produces an accepting proof by setting the challenges in each repetition to be the output of the least function of the first message 
and thus succeeds in passing the first test and hence the second test too. We now give details of the construction below:

Let $(P, V)$ be a Fiat-Shamir proof of knowledge for a relation $W$. We use two main tools for our construction, namely, a CPA symmetric encryption scheme $E=($ KeyGen, Enc, Dec) and a pseudorandom function family $\mathcal{F}$. Before we describe the protocol, we make the following assumption: In all the executions of the protocol, the prover and the verifier have access to a string which is generated by a Setup algorithm. The Setup algorithm takes $1^{k}$ as input and executes KeyGen to obtain SK. It further chooses a key $\mathrm{K}$ uniformly at random to choose a function from the pseudorandom function family $\mathcal{F}$. Finally, (SK, K) is output by Setup 3 The output of the Setup is used in the following way. Each time the prover or the verifier needs to encrypt a message $m$, they proceed as follows. Compute $f_{\mathrm{K}}(m)$ (where $f_{\mathrm{K}}$ is the function in $\mathcal{F}$ corresponding to key $\mathrm{K}$ ) to obtain $r$. To encrypt $m$, execute the algorithm Enc with inputs $m$, SK and $r$. Unless explicitly mentioned, by $\operatorname{Enc}(m)$ we mean that $m$ is encrypted using key SK and randomness $f_{\mathrm{K}}(m)$. This means that $\operatorname{Enc}(m)$ gives the same ciphertext every time it is executed. If we intend to use a different randomness, we use the notation $\operatorname{Enc}(m: R)$ to mean that $m$ is encrypted using the randomness $R$. When the prover or the verifier wants to decrypt a message $m$ they execute Dec with input $m$ and key SK. Jumping ahead, we need to encrypt messages because the hash function used to instantiate the random oracle might have the code of the verifier $V$ embedded in it. In this case the hash function may output values depending whether the input transcripts are accepting or rejecting. To make our security proof go through we need to make sure the hash function does not have the capability to distinguish the transcripts. We can ensure this by encrypting the messages of the prover (The Setup algorithm is considered to be a part of the interaction between a specific prover and a verifier; the hash function used to instantiate the random oracle is independent of the output of the Setup algorithm). We now proceed to describe the protocol.

As discussed before, we will first consider a Fiat-Shamir proof of knowledge $(P, V)$. We assume that the prover $P$ in $(P, V)$ can be decomposed into $P_{0}$ and $P_{1}$, where $P_{0}(x, w)$ outputs the commitment $\alpha$ and $P_{1}(x, w, \alpha, \beta)$ outputs $\gamma$. Similarly, the verifier $V$ can be decomposed into $V_{0}$ and $V_{1}$ such that $V_{0}$ interacts with $P$ (by outputting $\beta$ on input some $(x, \alpha))$ to produce the transcript $(\alpha, \beta, \gamma)$ and then $V$ accepts if and only if $V_{1}(\alpha, \beta, \gamma)$ accepts. We use the symbols $r, b, t$ as defined in the Fischlin construction (c.f. Section 2). We denote the least significant $l$ bits of $M(\boldsymbol{y})$ by $M(\boldsymbol{y})^{(l)}$.

Our protocol is parameterized by a polynomial $p_{\text {hash. }}$. We are now ready to describe the protocol $\left(P^{*}, V^{*}\right)$ for the relation $W$.

\section{Protocol $\left(P^{*}, V^{*}\right)$ :}

${ }^{3}$ We note that neither the prover nor the verifier needs to place any trust in the setup algorithm for their security. The reason to have (SK, K) as the public parameters (as opposed to the part of protocol messages) will become clear later on. 
1. $P^{*}$ : Run $P_{0}$ on $(x, w)$ to obtain $\alpha$. Define $\alpha^{*}=\operatorname{Enc}((\alpha, i$, bit, $\mathrm{M}))$, where each of $i$, bit, $\mathrm{M}$ is set to 0 , with their lengths being $\log (r)$ bits, 1 bit, and $|x|$ bits, respectively 4 . Send $\alpha^{*}$ to $V^{*}$.

[Note: Looking ahead, in the protocol obtained by first applying Fischlin transformation to $\left(P^{*}, V^{*}\right)$ and then instantiating it with the hash function, the adversary will set $i$ to be the repetition number, bit to be 1 and $M$ to be the hash function instantiating the random oracle.]

2. $V^{*}$ : Execute $\operatorname{Dec}\left(\alpha^{*}\right)$ to obtain $\alpha_{1}$ which is then parsed as $(\alpha, i$, bit, $M)$. Run $V_{0}$ on input $(x, \alpha)$ to obtain $\beta$. Send $\beta$ to $P^{*}$.

3. $P^{*}$ : Run $P_{1}$ on input $(x, w, \alpha, \beta)$ to obtain $\gamma$. Send $\gamma^{*}=\operatorname{Enc}(\gamma)$ to $V^{*}$.

$V^{*}$ then decides to accept or reject the transcript $\left(x, \alpha^{*}, \beta, \gamma^{*}\right)$ by executing the following.

i. Let $\alpha_{1} \leftarrow \operatorname{Dec}\left(\alpha^{*}\right)$ and $\gamma \leftarrow \operatorname{Dec}\left(\gamma^{*}\right)$.

ii. Parse $\alpha_{1}$ to be $(\alpha, i$, bit, $\mathrm{M})$.

iii. If bit $=0$ then Accept if and only if $V_{0}(x, \alpha, \beta, \gamma)$ accepts and $\gamma^{*}=\operatorname{Enc}(\gamma)$. [Note: Recall that $\operatorname{Enc}(m)$ is the encryption of $m$ using the randomness $f_{\mathrm{K}}(m)$. Hence, the check $\gamma^{*}=\operatorname{Enc}(\gamma)$ ensures that $\gamma^{*}$ is indeed the encryption of $\gamma$ using the randomness $f_{\mathrm{K}}(\gamma)$. Looking ahead, this will be helpful to make the protocol satisfy the unique responses property.]

iv. Else, do the following. If $\mathrm{M}$ is not a valid Turing machine then Reject. Otherwise, Accept if both the following conditions hold:

- $\beta=\operatorname{least}(x, \alpha, i, \mathrm{M})$.

- $\gamma^{*}=\operatorname{Enc}((i, \beta))$.

where the least procedure is defined below.

least $(x, \alpha, i, \mathrm{M})$ :

1. $\min \leftarrow 2^{b}+1$

2. $\beta \leftarrow$ null

3. For $j=0$ to $2^{t}-1$ :

4. $\boldsymbol{y} \leftarrow(x,(\operatorname{Enc}((\alpha, 1,1, \mathrm{M})), \ldots, \operatorname{Enc}((\alpha, r, 1, \mathrm{M})), i, j, \operatorname{Enc}((i, j))))$

5. Execute $\mathbf{M}(\boldsymbol{y})$ upto $p_{\text {hash }}(|\boldsymbol{y}|)$ steps

6. If $\mathrm{M}(\boldsymbol{y})$ terminates within $p_{\text {hash }}(|\boldsymbol{y}|)$ steps:

7. $\quad$ hash $\leftarrow \mathrm{M}(\boldsymbol{y})^{(b)}$

8. If $\min >$ hash:

9. $\quad \min \leftarrow$ hash

10. $\quad \beta \leftarrow j$

11. Return $\beta$.

${ }^{4}$ Hereafter, unless specified otherwise, we maintain that the lengths of bit, $i$ are specified above, and in every instance where we set $\mathrm{M}$ to 0 , it is of length $|x|$. 
The least algorithm does the following. It checks for what values of $j$ from 0 to $2^{t}-1$, the last $b$ bits of $\mathrm{M}(x,(\operatorname{Enc}((\alpha, 1,1, \mathrm{M})), \ldots, \operatorname{Enc}((\alpha, r, 1, \mathrm{M})), i, j, \operatorname{Enc}((i, j)))$ takes the minimum value among all possible $2^{t}$ values provided $\mathrm{M}$ terminates within $p_{\text {hash }}(|x, \operatorname{Enc}((\alpha, 1,1, \mathrm{M})), \ldots, \operatorname{Enc}((\alpha, r, 1, \mathrm{M})), i, j, \operatorname{Enc}((i, j))|)$ steps. If there are many values of $j$ for which the hash function maps to the minimum then it picks the one which is the smallest. Observe that in the Fischlin construction, the non-interactive prover $P^{\mathcal{O}}$ would implicitly run the least algorithm as follows. It rewinds the prover in the Fiat-Shamir proof of knowledge until it finds the smallest $\beta$ such that the hash value when applied on the entire transcript maps to a minimum. This observation was the main intuition behind our definition of the least algorithm.

We show that $\left(P^{*}, V^{*}\right)$ satisfies all the properties of Fiat-Shamir proof of knowledge.

Lemma 1. $\left(P^{*}, V^{*}\right)$ is a Fiat-Shamir proof of knowledge for the relation $W$.

Proof. Before we show that $\left(P^{*}, V^{*}\right)$ satisfies all the properties of Fiat-Shamir proof of knowledge, we first make the observation that both the prover $P^{*}$ and the verifier $V^{*}$ run in polynomial time.

[Completeness]. For any $\left(\alpha^{*}, \beta, \gamma^{*}\right)$ resulting from the interaction between $P^{*}$ and $V^{*}$ on input $x$, we have that $\alpha^{*}=\operatorname{Enc}((\alpha, i$, bit, $\mathrm{M}))$ and $\gamma^{*}=\operatorname{Enc}(\gamma)$ with each of $i$, bit, $\mathrm{M}$ being $0 . V^{*}$ accepts $\left(\alpha^{*}, \beta, \gamma^{*}\right)$ only if $V(x, \alpha, \beta, \gamma)$ accepts. Thus, the completeness of $\left(P^{*}, V^{*}\right)$ follows from the completeness property of $(P, V)$.

[Special Soundness]. Let $K$ be a knowledge extractor for $(P, V)$. We show that $\left(P^{*}, V^{*}\right)$ satisfies special soundness by constructing a knowledge extractor $K^{*}$ that uses $K$ as follows. For any $(x, w) \in W$, on input $\left(x, \alpha^{*}, \beta_{1}, \gamma_{1}^{*}, \beta_{2}, \gamma_{2}^{*}\right)$ such that $V^{*}\left(x, \alpha^{*}, \beta_{1}, \gamma_{1}^{*}\right)=V^{*}\left(x, \alpha^{*}, \beta_{2}, \gamma_{2}^{*}\right)=$ Accept and $\beta_{1} \neq \beta_{2}, K^{*}$ does the following. It decrypts $\alpha^{*}$ to obtain $\left(\alpha, i\right.$, bit, M). Similarly it decrypts $\gamma_{1}^{*}$ and $\gamma_{2}^{*}$ to obtain $\gamma_{1}$ and $\gamma_{2}$ respectively. Then, $K^{*}$ outputs whatever $K\left(x, \alpha, \beta_{1}, \gamma_{1}, \beta_{2}, \gamma_{2}\right)$ outputs. To see that $K^{*}$ is indeed a knowledge extractor for $\left(P^{*}, V^{*}\right)$, consider the following two cases.

- bit $=0$ : Here, $V^{*}\left(x, \alpha^{*}, \beta_{1}, \gamma_{1}^{*}\right)=V^{*}\left(x, \alpha^{*}, \beta_{2}, \gamma_{2}^{*}\right)=$ Accept only if $V\left(x, \alpha, \beta_{1}, \gamma_{1}\right)=V\left(x, \alpha, \beta_{2}, \gamma_{2}\right)=$ Accept. Hence the special soundness property is satisfied because the special soundness of $(P, V)$ ensures that for such an input $\left(x, \alpha, \beta_{1}, \gamma_{1}, \beta_{2}, \gamma_{2}\right), K$ outputs $w^{\prime}$ such that $\left(x, w^{\prime}\right) \in W$.

- bit $=1$ : In this case, $V^{*}$ accepts both inputs $\left(\alpha^{*}, \beta_{1}, \gamma_{1}^{*}\right)$ and $\left(\alpha^{*}, \beta_{2}, \gamma_{2}^{*}\right)$ only if both $\beta_{1}$ and $\beta_{2}$ are equal to least $(\alpha, i, 1, \mathrm{M})$. This contradicts the assumption that $\beta_{1} \neq \beta_{2}$.

[Commitment entropy]. The first message of $P^{*}$ contains $\alpha$ which has the same distribution as the first message of $P$ and hence the commitment entropy property is satisfied. 
[Public coin]. This follows from the description of $V^{*}$.

[Unique responses]. For any probabilistic polynomial-time algorithm $\mathcal{A}$ and $\left(x, \alpha^{*}, \beta, \gamma_{1}^{*}, \gamma_{2}^{*}\right) \leftarrow \mathcal{A}\left(1^{k}\right)$, where $\alpha^{*}=\operatorname{Enc}((\alpha, i, \operatorname{bit}, \mathrm{M})), \operatorname{Enc}\left(\gamma_{1}\right)=\gamma_{1}^{*}$ and $\operatorname{Enc}\left(\gamma_{2}\right)=\gamma_{2}^{*}$. We claim that the following is negligible in $k$ :

$$
\operatorname{Pr}\left[V^{*}\left(\alpha^{*}, \beta, \gamma_{1}^{*}\right)=V^{*}\left(\alpha^{*}, \beta, \gamma_{2}^{*}\right)=\text { Accept \& } \gamma_{1}^{*} \neq \gamma_{2}^{*}\right] .
$$

To prove this claim, consider the following cases.

- bit $=0$ : Observe that in this case, $V^{*}\left(x, \alpha^{*}, \beta, \gamma_{1}^{*}\right)=$ Accept only if $V\left(x, \alpha, \beta, \gamma_{1}\right)=$ Accept, and $V^{*}\left(x, \alpha^{*}, \beta, \gamma_{2}^{*}\right)=$ Accept only if $V\left(x, \alpha, \beta, \gamma_{2}\right)=$ Accept. Also, $\gamma_{1}$ is equal to $\gamma_{2}$ only if $\gamma_{1}^{*}$ is equal to $\gamma_{2}^{*}$. This is because of the following reason. $\gamma_{1}^{*}$ is the encryption of $\gamma_{1}$ using the randomness $f_{K}\left(\gamma_{1}\right)$ and $\gamma_{2}^{*}$ is the encryption of $\gamma_{2}$ using the randomness $f_{\mathrm{K}}\left(\gamma_{2}\right)$. And hence if $\gamma_{1}$ were to be equal to $\gamma_{2}$ then this would imply that $\gamma_{1}^{*}$ equals $\gamma_{2}^{*}$. Combining the above arguments we have the following. Conditioned on bit $=0$,

$$
\begin{gathered}
\operatorname{Pr}\left[V^{*}\left(\alpha^{*}, \beta, \gamma_{1}^{*}\right)=V^{*}\left(\alpha^{*}, \beta, \gamma_{2}^{*}\right)=\text { Accept \& } \gamma_{1}^{*} \neq \gamma_{2}^{*}\right] \\
=\operatorname{Pr}\left[V\left(\alpha, \beta, \gamma_{1}\right)=V\left(\alpha, \beta, \gamma_{2}\right)=\text { Accept \& } \gamma_{1} \neq \gamma_{2}\right]
\end{gathered}
$$

Since $(P, V)$ satisfies the unique responses property, the quantity $\operatorname{Pr}\left[V\left(\alpha, \beta, \gamma_{1}\right)=V\left(\alpha, \beta, \gamma_{2}\right)=\right.$ Accept $\left.\& \gamma_{1} \neq \gamma_{2}\right]$ is negligible and hence the claim follows.

- bit $=1$ : Note that in this case, one of the conditions that needs to be satisfied for $V^{*}$ to accept $\left(\alpha^{*}, \beta, \gamma_{1}^{*}\right)$ (and $\left.\left(\alpha^{*}, \beta, \gamma_{2}^{*}\right)\right)$ is that $\gamma_{1}^{*}=\operatorname{Enc}((i, \beta))$ (resp., $\left.\gamma_{2}^{*}=\operatorname{Enc}((i, \beta))\right)$. This implies that $\gamma_{1}^{*}=\gamma_{2}^{*}$ and hence the following holds conditioned on bit $=1$.

$$
\operatorname{Pr}\left[V^{*}\left(\alpha^{*}, \beta, \gamma_{1}^{*}\right)=V^{*}\left(\alpha^{*}, \beta, \gamma_{2}^{*}\right)=\text { Accept } \& \gamma_{1}^{*} \neq \gamma_{2}^{*}\right]=0
$$

[Honest Verifier Zero-knowledge]. To prove that $\left(P^{*}, V^{*}\right)$ satisfies honest verifer zero-knowledge property, we construct a zero-knowledge simulator $Z^{*}$ for $\left(P^{*}, V^{*}\right)$ as follows. Let $Z$ be a zero-knowledge simulator for the protocol $(P, V)$. On input (x,membership), where membership $\in\{$ yes, no $\}, Z^{*}$ runs $Z\left(x\right.$, membership) to obtain $(\alpha, \beta, \gamma) . Z^{*}$ outputs $(\operatorname{Enc}((\alpha, i, \operatorname{bit}, \mathrm{M})), \beta$, Enc $(\gamma))$, where $i$, bit and $\mathrm{M}$ are set to 0 .

To prove that $Z^{*}$ is a zero-knowledge simulator for $\left(P^{*}, V^{*}\right)$, we first assume for contradiction that there exists a distinguisher $D^{*}=\left(D_{0}^{*}, D_{1}^{*}\right)$ such that the statistical distance between following two distributions is non-negligible.

- Distreal : Let $(x, w$, state $) \leftarrow D_{0}^{*}\left(1^{k}\right)$ and $\left(\alpha^{*}, \beta, \gamma^{*}\right) \leftarrow\left(P^{*}(w), V^{*}\right)(x)$ if $(x, w) \in W$, and $\left(\alpha^{*}, \beta, \gamma^{*}\right) \leftarrow \perp$ otherwise. Output $D_{1}^{*}\left(\alpha^{*}, \beta, \gamma^{*}\right.$, state).

- Dist sim: Let $(x, w$, state $) \leftarrow D_{0}^{*}\left(1^{k}\right)$ and $\left(\alpha^{*}, \beta, \gamma^{*}\right) \leftarrow Z^{*}(x$, yes $)$ if $(x, w) \in$ $W$, and $\left(\alpha^{*}, \beta, \gamma^{*}\right) \leftarrow Z^{*}(x$, no $)$ otherwise. Output $D_{1}^{*}\left(\alpha^{*}, \beta, \gamma^{*}\right.$, state $)$. 
Then, we construct a distinguisher $D=\left(D_{0}, D_{1}\right)$ that contradicts the honest verifier zero-knowledge property of $(P, V)$ as follows. $D_{0}\left(1^{k}\right)$ runs $D_{0}^{*}\left(1^{k}\right)$ to obtain $(x, w$, state $)$ and outputs the same. Once $D_{1}$ receives $(\alpha, \beta, \gamma)$, if $(\alpha, \beta, \gamma) \neq \perp$ then it outputs $D_{1}^{*}(\operatorname{Enc}((\alpha, i$, bit, $\mathrm{M})), \beta$, Enc $(\gamma)$, state $)$, where $i$, bit and $\mathrm{M}$ are set to 0 , else it outputs $D_{1}^{*}(\perp$, state). Now consider the following distributions.

- Dist $_{\text {real }}$ : Let $(x, w$, state $) \leftarrow D_{0}\left(1^{k}\right)$ and $(\alpha, \beta, \gamma) \leftarrow(P(w), V)(x)$ if $(x, w) \in$ $W$, and $(\alpha, \beta, \gamma) \leftarrow \perp$ otherwise. Output $D_{1}(\alpha, \beta, \gamma$, state).

- Dist sim $_{\text {: Let }}(x, w$, state $) \leftarrow D_{0}\left(1^{k}\right)$ and $(\alpha, \beta, \gamma) \leftarrow Z(x$, yes $)$ if $(x, w) \in W$, and $(\alpha, \beta, \gamma) \leftarrow Z(x$, no $)$ otherwise. Output $D_{1}(\alpha, \beta, \gamma$, state $)$.

Now, from the way $Z^{*}$ and $D$ are constructed, the distribution Dist real $_{\text {is }}$ the same as Dist ${ }_{\text {real }}^{*}$. Similarly, the distribution Dist ${ }_{\text {sim }}$ is the same as Dist ${ }_{\text {sim }}^{*}$. This implies that the statistical distance between Dist $_{\text {real }}$ and Dist ${ }_{\text {sim }}$ is also non-negligible, a contradiction.

\subsection{On the Insecurity of $\left(P_{h}, V_{h}\right)$}

The non-interactive zero-knowledge proof of knowledge $\left(P^{* \mathcal{O}}, V^{* \mathcal{O}}\right)$ obtained by applying the Fischlin transformation to $\left(P^{*}, V^{*}\right)$ is sound in the random oracle model. This follows from the fact that $\left(P^{*}, V^{*}\right)$ is Fiat-Shamir proof of knowledge (Theorem 3) and any protocol obtained by applying Fischlin transformation to a Fiat-Shamir proof of knowledge is secure in the random oracle model. In this section, we show that when the random oracle is instantiated by a hash function $h$, whose worst case running time is at most the polynomial $p_{\text {hash }}$ in the size of its inputs, the protocol $\left(P_{h}, V_{h}\right)$, which is obtained by instantiating the random oracle $\mathcal{O}$ in $\left(P^{* \mathcal{O}}, V^{* \mathcal{O}}\right)$, is not sound. Typically, $p_{\text {hash }}$ is chosen to be a polynomial of degree $c$, for a large constant $c$. The following theorem rules out the secure instantiation of the Fischlin construction with most of the practical hash functions.

Theorem 1. Let $\left(P^{*}, V^{*}\right)$ be the 3-round Fiat-Shamir proof of knowledge, for a witness relation $W$ and the corresponding language $L=\{x:(x, w) \in W\}$, as described above. Let $\left(P^{* \mathcal{O}}, V^{* \mathcal{O}}\right)$ be the non-interactive zero-knowledge proof of knowledge obtained by applying the Fischlin transformation to $\left(P^{*}, V^{*}\right)$. Then, for any hash function $h$, that is used to instantiate the random oracle $\mathcal{O}$, and whose running time is at most $p_{\text {hash }}(|\boldsymbol{y}|)$ for any input $\boldsymbol{y} \in\{\mathbf{0 , 1}\}^{*}$, the resulting protocol $\left(P_{h}, V_{h}\right)$ is not sound. In other words, there exists an adversary $\mathcal{A}$ such that $\operatorname{Pr}\left[(x, \pi) \leftarrow A\left(1^{k}\right): V^{h}(x, \pi)=1\right.$ and $\left.x \notin L\right]$ is non-negligible.

The proof of the above theorem can be found in the full version.

\section{Simplified Construction for Pseudorandom Hash Functions}

In this section, we present a simpler construction to demonstrate the insecurity of the Fischlin transformation with respect to hash functions which behave as 
pseudorandom functions (i.e., the output of such a hash function for any input is indistinguishable from random). As in the main construction, we restrict our attention to the case when the worst case running time of the hash function is at most some fixed polynomial in the size of the input. More formally, the insecurity arguments hold only for those hash functions whose running time is at most $p_{\text {hash }}(|\boldsymbol{y}|)$ on input $\boldsymbol{y}$, where $p_{\text {hash }}$ is a polynomial. Unlike the main construction, the construction presented below does not require any initial setup.

Let $(P, V)$ be any 3-round Fiat-Shamir proof of knowledge for some witness relation $W$. We extend $(P, V)$ to obtain a 3 -round Fiat-Shamir proof of knowledge $\left(P^{*}, V^{*}\right)$ as we shall describe shortly.

Let $P=\left(P_{0}, P_{1}\right)$ and $V=\left(V_{0}, V_{1}\right)$. On input $(x, w), P_{0}$ generates $\alpha$, and on input $(x, w, \alpha, \beta) P_{1}$ generates $\gamma$. Also, $V_{0}$ and $V_{1}$ are such that $V_{0}$ interacts with $P$ to produce a transcript $(\alpha, \beta, \gamma)$ by generating $\beta$ uniformly at random and then $V$ accepts if and only if $V_{1}(x, \alpha, \beta, \gamma)$ accepts. The protocol $\left(P^{*}, V^{*}\right)$ is described below.

1. $P^{*}$ : Run $P_{0}$ on $(x, w)$ to obtain $\alpha$. Define $\alpha^{*}=(\alpha, i$, bit, $\mathrm{M})$, where each of $i$, bit, $\mathrm{M}$ is set to 0 , with their lengths being $\log (r)$ bits, 1 bit, and and $|x|$ bits, respectively 5 . Send $\alpha^{*}$ to $V^{*}$.

2. $V^{*}$ : Run $V_{0}$ to obtain $\beta$. Send $\beta$ to $P^{*}$.

3. $P^{*}$ : Run $P_{1}$ on input $(x, w, \alpha, \beta)$ to obtain $\gamma$. Send $\gamma$ to $V^{*}$.

4. $V^{*}$ : Parse $\alpha^{*}$ as $\left(\alpha, i\right.$, bit, M). If bit $=0$ then Accept if $V_{0}(x, \alpha, \beta, \gamma)$ accepts. If bit $=1$ then check if $M$ is a valid Turing Machine. If $M$ is not a valid TM then Reject. Else, Accept if all the following conditions hold:

- $\beta=$ least $(x, \alpha, i, 1, \mathrm{M})$

- $\gamma=0$

where the function least is defined as follows.

least $(x, \alpha, i, \mathrm{M})$ :

1. $\min \leftarrow 2^{b}+1$

2. $\beta \leftarrow$ null

3. For $j=0$ to $2^{t}-1$ :

4. $\boldsymbol{y} \leftarrow(x,((\alpha, 1,1, \mathrm{M}), \ldots,(\alpha, r, 1, \mathrm{M})), i, j, 0)$

5. Execute $\mathbf{M}(\boldsymbol{y})$ upto $p_{\text {hash }}(|\boldsymbol{y}|)$ steps

6. If $\mathrm{M}(\boldsymbol{y})$ terminates within $p_{\text {hash }}(|\boldsymbol{y}|)$ steps:

7. hash $\leftarrow \mathrm{M}(\boldsymbol{y})^{(b)}$

8. If $\min >$ hash:

9. $\quad \min \leftarrow$ hash

10. $\quad \beta \leftarrow j$

11. Return $\beta$.

${ }^{5}$ Hereafter, unless specified otherwise, we maintain that the lengths of bit, $i, \mathrm{M}$ are as specified here. 
The functionality of the least algorithm defined above is very similar to the one defined in the main construction with the main difference being that the first message and the last messages are not encrypted in the algorithm described above unlike the least algorithm defined in the main construction. Further, the symbols $r, b, t$ are as described in the Fischlin construction (c.f. Section 2). We now show that $\left(P^{*}, V^{*}\right)$ satisfies all the properties of Fiat-Shamir proof of knowledge.

\subsection{On the Security of $\left(P^{*}, V^{*}\right)$}

Theorem 2. $\left(P^{*}, V^{*}\right)$ is a Fiat-Shamir proof of knowledge for the relation $W$.

Proof. [Completeness] For any $\left(\alpha^{*}, \beta, \gamma\right)$ resulting from the interaction between $P^{*}$ and $V^{*}$, we have that $\alpha^{*}=(\alpha, i$, bit, M ) with each of $i$, bit, M being 0 and with $(\alpha, \beta, \gamma)$ generated as per the description of $(P, V)$. Since, if $V$ accepts $(\alpha, \beta, \gamma)$ then $V^{*}$ accepts $\left(\alpha^{*}, \beta, \gamma\right)$, completeness of $(P, V)$ implies that of $\left(P^{*}, V^{*}\right)$.

[Special Soundness]. Let $K$ be a knowledge extractor for $(P, V)$. We show that $\left(P^{*}, V^{*}\right)$ satisfies special soundness by constructing a knowledge extractor $K^{*}$ that uses $K$ as follows: For any $(x, w) \in W_{k}$, on input $\left(x, \alpha^{*}, \beta, \gamma, \beta^{\prime}, \gamma^{\prime}\right)$, where $\alpha^{*}=(\alpha, i$, bit, $\mathrm{M}), \beta \neq \beta^{\prime}$, and $V^{*}(x,(\alpha, i$, bit, $\mathrm{M}), \beta, \gamma)=V^{*}(x,(\alpha, i$, bit, $\mathrm{M})$, $\left.\beta^{\prime}, \gamma^{\prime}\right)=$ Accept, $K^{*}$ outputs $K\left(x, \alpha, \beta, \gamma, \beta^{\prime}, \gamma^{\prime}\right)$. To see that $K^{*}$ is indeed a knowledge extractor for $\left(P^{*}, V^{*}\right)$, consider the following two cases.

- bit $=0$ : Here, $V^{*}(x,(\alpha, i$, bit, M $), \beta, \gamma)=V^{*}\left(x,(\alpha, i\right.$, bit, M $\left.), \beta^{\prime}, \gamma^{\prime}\right)=$ Accept implies that $V(x, \alpha, \beta, \gamma)=V\left(x, \alpha, \beta^{\prime}, \gamma^{\prime}\right)=$ Accept. Since special soundness of $(P, V)$ ensures that for an input $\left(x, \alpha, \beta, \gamma, \beta^{\prime}, \gamma^{\prime}\right), K$ outputs $w^{\prime}$ such that $\left(x, w^{\prime}\right) \in W_{k}, K^{*}$ also outputs a witness.

- bit $=1$ : In this case, $V^{*}$ accepts on both inputs $((\alpha, i$, bit, M), $\beta, \gamma)$ and $\left((\alpha, i\right.$, bit, $\left.\mathrm{M}), \beta^{\prime}, \gamma^{\prime}\right)$ only if both $\beta$ and $\beta^{\prime}$ are equal to least $(\alpha, i, 1, \mathrm{M})$ which leads to a contradiction to $\beta \neq \beta^{\prime}$.

[Commitment entropy]. The first message of $P^{*}$ contains $\alpha$ which has the same distribution as the first message of $P$ and hence the commitment entropy property is satisfied.

[Public coin]. This follows directly from the description of $V^{*}$.

[Unique responses]. For any probabilistic polynomial-time algorithm $\mathcal{A}$, and $\left(x,(\alpha, i\right.$, bit, $\left.\mathrm{M}), \beta, \gamma, \gamma^{\prime}\right) \leftarrow \mathcal{A}\left(1^{k}\right)$, we claim that the following is negligible in $k$ :

$$
\operatorname{Pr}\left[V^{*}\left((\alpha, i \text {, bit, M) }, \beta, \gamma)=V^{*}\left((\alpha, i, \text { bit, } \mathrm{M}), \beta, \gamma^{\prime}\right)=\text { Accept \& } \gamma \neq \gamma^{\prime}\right]\right. \text {. }
$$

We establish the claim under the following two cases.

- bit $=0:$ Note that, $V^{*}(x,(\alpha, i$, bit, M $), \beta, \gamma)=$ Accept implies that $V(x, \alpha, \beta, \gamma)=$ Accept, and also, $V^{*}\left(x,(\alpha, i\right.$, bit, M $\left.), \beta, \gamma^{\prime}\right)=$ Accept implies that $V\left(x, \alpha, \beta, \gamma^{\prime}\right)=$ Accept. Since $\operatorname{Pr}\left[V(\alpha, \beta, \gamma)=V\left(\alpha, \beta, \gamma^{\prime}\right)=\right.$ Accept \& $\left.\gamma \neq \gamma^{\prime}\right]$ is negligibly close to 0 , we have $\operatorname{Pr}\left[V^{*}((\alpha, i\right.$, bit, $h), \beta, \gamma)=$ $V^{*}\left((\alpha, i\right.$, bit,$\left.h), \beta, \gamma^{\prime}\right)=$ Accept $\& \gamma \neq \gamma^{\prime} \mid$ bit $\left.=0\right]$ is negligibly close to 0 . 
- bit $=1$ : Observe that, $V^{*}(x,(\alpha, i$, bit, M), $\beta, \gamma)=$ Accept implies that $\gamma=0$, and also, $V^{*}\left(x,(\alpha, i\right.$, bit, M $\left.), \beta, \gamma^{\prime}\right)=$ Accept implies that $\gamma=0$, thus giving us $\gamma=\gamma^{\prime}$.

[Honest Verifier Zero-knowledge]. To prove that $\left(P^{*}, V^{*}\right)$ is an HVZK protocol, we construct a special zero-knowledge simulator $Z^{*}$ for $\left(P^{*}, V^{*}\right)$ as follows. Let $Z$ be a special zero-knowledge simulator for the protocol $(P, V)$. On input $\left(x, \beta\right.$, memebership), where memebership $\in\{$ yes, no $\}, Z^{*}$ runs $Z\left(x, \beta\right.$, memebership) to obtain $(\alpha, \beta, \gamma)$. If $(\alpha, \beta, \gamma)=\perp$, then $Z^{*}$ also outputs $\perp$; otherwise, it outputs $((\alpha, i$, bit, M), $\beta, \gamma)$, where $i$, bit and $\mathrm{M}$ are set to 0 .

To prove that $Z^{*}$ is a special zero-knowledge simulator for $\left(P^{*}, V^{*}\right)$, assume for contradiction that there exists a distinguisher $D^{*}=\left(D_{0}^{*}, D_{1}^{*}\right)$ such that the statistical distance, $\epsilon(k)$, between following two distributions is non-negligible.

- Dist real $^{*}$ : Let $(x, w$, state $) \leftarrow D_{0}^{*}\left(1^{k}\right)$ and $\left(\alpha^{*}, \beta, \gamma\right) \leftarrow\left(P^{*}(w), V^{*}\right)(x)$ if $(x, w) \in W_{k}$, and $\left(\alpha^{*}, \beta, \gamma\right) \leftarrow \perp$ otherwise. Output $D_{1}^{*}\left(\alpha^{*}, \beta, \gamma\right.$, state).

- Dist sim : Let $(x, w$, state $) \leftarrow D_{0}^{*}\left(1^{k}\right)$ and $\left(\alpha^{*}, \beta, \gamma\right) \leftarrow Z^{*}(x$, yes $)$ if $(x, w) \in W_{k}$, and $\left(\alpha^{*}, \beta, \gamma\right) \leftarrow Z^{*}(x$, no $)$ otherwise. Output $D_{1}^{*}\left(\alpha^{*}, \beta, \gamma\right.$, state).

Then, we construct a distinguisher $D=\left(D_{0}, D_{1}\right)$ against the HVZK property of $(P, V)$ as follows. $D_{0}\left(1^{k}\right)$ runs $D_{0}^{*}\left(1^{k}\right)$ to obtain $(x, w$, state $)$ and outputs the same. Once $D_{1}$ receives $(\alpha, \beta, \gamma)$, if $(\alpha, \beta, \gamma) \neq \perp$ then it outputs $D_{1}^{*}((\alpha, i$, bit, $\mathrm{M}), \beta, \gamma$, state), where $i$, bit and $\mathrm{M}$ are set to 0 ; otherwise, it sets $\left(\alpha^{*}, \beta, \gamma\right) \leftarrow \perp$ and outputs $D_{1}^{*}\left(\alpha^{*}, \beta, \gamma\right.$, state). Now consider the following distributions.

- Dist real $_{\text {: Let }}(x, w$, state $) \leftarrow D_{0}\left(1^{k}\right)$ and $(\alpha, \beta, \gamma) \leftarrow(P(w), V)(x)$ if $(x, w) \in$ $W_{k}$, and $(\alpha, \beta, \gamma) \leftarrow \perp$ otherwise. Output $D_{1}(\alpha, \beta, \gamma$, state).

- Dist sim $_{\text {sim }}$ Let $(x, w$, state $) \leftarrow D_{0}\left(1^{k}\right)$ and $(\alpha, \beta, \gamma) \leftarrow Z$ (x, yes) if $(x, w) \in W_{k}$, and $(\alpha, \beta, \gamma) \leftarrow Z(x$, no $)$ otherwise. Output $D_{1}\left(\alpha^{*}, \beta, \gamma\right.$, state).

Now, from the way $Z^{*}$ and $D$ are constructed, the output of Dist real $_{\text {is }}$ the same as the output of Dist real. Similarly, the output of Dist sim $_{\text {im }}^{*}$ is the same as the output of Dist ${ }_{\text {sim }}^{*}$. This implies that the statistical distance between Dist ${ }_{\text {real }}$ and Dist sim $_{\text {sim }}$ is also $\epsilon(k)$, a contradiction.

\subsection{On the Insecurity of $\left(P_{h}, V_{h}\right)$}

Let $\mathcal{F}_{p_{\text {hash }}}$ be a pseudorandom function family such that each function in the family can be be evaluated on any input in time $p_{\text {hash }}$ in the size of its inputs, where $p_{\text {hash }}$ is a polynomial. Now we shall show that for every such hash function that is used to instantiate the random oracle in the non-interactive ZK PoK that is obtained by applying Fischlin transformation to $\left(P^{*}, V^{*}\right)$, the resulting protocol does not satisfy the soundness.

In order to model a pseudorandom hash function family, one may look at the function to take a randomly chosen secret key also as an input. Thus, to instantiate the random oracle, first a key $K$ is picked uniformly at random. 
Let $f_{K}$ be the pseudorandom hash function corresponding to $K$ in $\mathcal{F}_{p_{\text {hash }}}$. Henceforth, we shall denote $f_{K}$ by $h$ for a simpler notation. Let $\left(P_{h}, V_{h}\right)$ be the protocol obtained by applying Fischlin transformation to $\left(P^{*}, V^{*}\right)$. The following theorem shows that the protocol $\left(P_{h}, V_{h}\right)$ does not satisfy the soundness property.

Theorem 3. Let $\left(P^{*}, V^{*}\right)$ be the 3-round Fiat-Shamir proof of knowledge described above for a witness relation $W$ with corresponding language $L$ and let $\left(P^{\mathcal{O}}, V^{\mathcal{O}}\right)$ be the non-interactive zero-knowledge proof of knowledge protocol obtained by applying Fischlin transformation to $\left(P^{*}, V^{*}\right)$. Then for any pseudorandom hash function $h$ in $\mathcal{F}_{p_{\text {hash }}}$ that is used to instantiate the random oracle $\mathcal{O}$, the resulting protocol $\left(P_{h}, V_{h}\right)$ does not satisfy soundness. In other words, there exists an adversary $\mathcal{A}$ that outputs $(x, \pi)$ such that $\operatorname{Pr}\left[V_{h}(x, \pi)=\right.$ Accept and $\left.x \notin L\right]$ is non-negligible.

The proof of the above theorem can be found in the full version.

\section{References}

[AABN02] Abdalla, M., An, J.H., Bellare, M., Namprempre, C.: From Identification to Signatures via the Fiat-Shamir Transform: Minimizing Assumptions for Security and Forward-Security. In: Knudsen, L.R. (ed.) EUROCRYPT 2002. LNCS, vol. 2332, pp. 418-433. Springer, Heidelberg (2002)

[Bar01] Barak, B.: How to go beyond the black-box simulation barrier. In: FOCS, pp. 106-115 (2001)

[BBS04] Boneh, D., Boyen, X., Shacham, H.: Short Group Signatures. In: Franklin, M. (ed.) CRYPTO 2004. LNCS, vol. 3152, pp. 41-55. Springer, Heidelberg (2004)

[BGGL01] Barak, B., Goldreich, O., Goldwasser, S., Lindell, Y.: Resettably-sound zero-knowledge and its applications. In: FOCS, pp. 116-125 (2001)

$\left[B G I^{+} 01\right]$ Barak, B., Goldreich, O., Impagliazzo, R., Rudich, S., Sahai, A., Vadhan, S.P., Yang, K.: On the (Im)possibility of Obfuscating Programs. In: Kilian, J. (ed.) CRYPTO 2001. LNCS, vol. 2139, pp. 1-18. Springer, Heidelberg (2001)

[BR93] Bellare, M., Rogaway, P.: Random oracles are practical: A paradigm for designing efficient protocols. In: ACM Conference on Computer and Communications Security, pp. 62-73 (1993)

[CGH04] Canetti, R., Goldreich, O., Halevi, S.: The random oracle methodology, revisited. J. ACM 51, 557-594 (2004)

[DGS09] Deng, Y., Goyal, V., Sahai, A.: Resolving the simultaneous resettability conjecture and a new non-black-box simulation strategy. In: FOCS, pp. 251-260 (2009)

[Fis05] Fischlin, M.: Communication-Efficient Non-interactive Proofs of Knowledge with Online Extractors. In: Shoup, V. (ed.) CRYPTO 2005. LNCS, vol. 3621, pp. 152-168. Springer, Heidelberg (2005)

[FS86] Fiat, A., Shamir, A.: How to Prove Yourself: Practical Solutions to Identification and Signature Problems. In: Odlyzko, A.M. (ed.) CRYPTO 1986. LNCS, vol. 263, pp. 186-194. Springer, Heidelberg (1987)

[GK03] Goldwasser, S., Kalai, Y.T.: On the (in)security of the fiat-shamir paradigm. In: FOCS, pp. 102-113 (2003) 
[GK05] Goldwasser, S., Kalai, Y.T.: Yael Tauman Kalai. On the impossibility of obfuscation with auxiliary input. In: 46th Annual IEEE Symposium on Foundations of Computer Science, FOCS 2005, pp. 553-562 (October 2005)

[GQ88] Guillou, L.C., Quisquater, J.-J.: A "Paradoxical" Identity-Based Signature Scheme Resulting from Zero-Knowledge. In: Goldwasser, S. (ed.) CRYPTO 1988. LNCS, vol. 403, pp. 216-231. Springer, Heidelberg (1990)

[MR02] Micali, S., Reyzin, L.: Improving the exact security of digital signature schemes. J. Cryptology 15(1), 1-18 (2002)

[Oka92] Okamoto, T.: Provably Secure and Practical Identification Schemes and Corresponding Signature Schemes. In: Brickell, E.F. (ed.) CRYPTO 1992. LNCS, vol. 740, pp. 31-53. Springer, Heidelberg (1993)

[Pas04] Pass, R.: Bounded-concurrent secure multi-party computation with a dishonest majority, pp. 232-241 (2004)

[PS00] Pointcheval, D., Stern, J.: Security arguments for digital signatures and blind signatures. J. Cryptology 13(3), 361-396 (2000)

[Sch91] Schnorr, C.-P.: Efficient signature generation by smart cards. J. Cryptology 4(3), 161-174 (1991) 